\title{
The Bogomolov multiplier of finite simple groups
}

\author{
Boris Kunyavskiu
}

\begin{abstract}
The subgroup of the Schur multiplier of a finite group $G$ consisting of all cohomology classes whose restriction to any abelian subgroup of $G$ is zero is called the Bogomolov multiplier of $G$. We prove that if $G$ is quasisimple or almost simple, its Bogomolov multiplier is trivial except for the case of certain covers of $P S L(3,4)$.
\end{abstract}

\section{Introduction}

A common method for proving that a given algebraic variety $X$ over a field $k$ is not rational is as follows. We consider some easily computable object (usually of algebraic nature), which can be defined functorially on a sufficiently large class of algebraic varieties and is known to be preserved under birational transformations (birational invariant). We calculate its value for $X$ (or for some $Y$ birationally equivalent to $X$ ). If this value is not trivial, i.e., does not coincide with the value of this birational invariant on the affine or projective space, $X$ is not rational.

The Brauer group $\operatorname{Br}(X)=H_{\text {èt }}^{2}\left(X, \mathbb{G}_{\mathrm{m}}\right)$, whose birational invariance in the class of smooth projective varieties has been established by Grothendieck, turned out to be a very convenient tool (the Artin-Mumford counter-example to Lüroth's problem, based on using this invariant, confirms its power). Moreover, even if $X$ is not projective, this invariant can be useful: embed $X$ as

Department of Mathematics Bar-Ilan University

52900 Ramat Gan, ISRAEL

kunyav@macs.biu.ac.il 
an open subset into a smooth projective variety $Y$ (if the ground field is of characteristic zero, this is always possible by Hironaka) and compute $\operatorname{Br}(Y)$. If the latter group is not zero, $Y$ cannot be birational to $\mathbb{P}^{n}$, and thus $X$ is not rational. Note that $\operatorname{Br}(Y)$ depends only on $X$ (and not on the choice of a smooth projective model $Y$ ); it is called the unramified Brauer group of $X$ and denoted by $\operatorname{Br}_{n r}(X)$. (The reader interested in historical perspective and geometric context, including more general invariants arising from higherdimensional cohomology, is referred to [Sh, [CTS, GS, 6.6, 6.7], Bo07].)

In concrete cases, it may be difficult to construct $Y$ explicitly, and thus it is desirable to express $\operatorname{Br}_{n r}(X)$ in intrinsic terms, i.e., get a formula not depending on $Y$. This approach was realized by Bogomolov [Bo87] in the case of the quotient variety $X=V / G$ where $V$ stands for a faithful linear representation of a linear algebraic group $G$ over $\mathbb{C}$. It turns out that in this case $\operatorname{Br}_{\mathrm{nr}}(X)$ depends solely on $G$ (but not on $V$ ).

In the present paper we focus on the case where $G$ is a finite group. The birational invariant $\operatorname{Br}_{n r}(V / G)$ has been used by Saltman to give a negative answer to Noether's problem [Sa]. In [Bo87] Bogomolov established an explicit formula for $\operatorname{Br}_{\mathrm{nr}}(V / G)$ in terms of $G$ : this group is isomorphic to $B_{0}(G)$, the subgroup of the Schur multiplier $\mathrm{M}(G):=H^{2}(G, \mathbb{Q} / \mathbb{Z})$ consisting of all cohomology classes whose restriction to any abelian subgroup of $G$ is zero. We call $B_{0}(G)$ the Bogomolov multiplier of $G$.

In [Sa], Bo87] one can find examples of groups $G$ with nonzero $B_{0}(G)$ (they are all $p$-groups of small nilpotency class). In contrast, in [Bo92] Bogomolov conjectured that $B_{0}(G)=0$ when $G$ is a finite simple group. In BMP] it was proved that $B_{0}(G)=0$ when $G$ is of Lie type $A_{n}$. In the present paper we prove Bogomolov's conjecture in full generality.

\section{Results}

We maintain the notation of the introduction and assume throughout the paper that $G$ is a finite group.

We say that $G$ is quasisimple if $G$ is perfect and its quotient by the centre $L=G / Z$ is a nonabelian simple group. We say that $G$ is almost simple if for some nonabelian simple group $L$ we have $L \subseteq G \subseteq$ Aut $L$. Our first observation is

Theorem 1.1. If $G$ is a finite quasisimple group other than a 4 -or 12-cover of $\operatorname{PSL}(3,4)$, then $B_{0}(G)=0$.

Corollary 1.2. If $G$ is a finite simple group, then $B_{0}(G)=0$. 
This corollary proves Bogomolov's conjecture.

From Corollary 1.2 we deduce the following

Theorem 1.3. If $G$ is a finite almost simple group, then $B_{0}(G)=0$.

Remark 1.4. Following GL, Ch. $2, \S \S 6,7]$, we call quasisimple groups $Q$ (as in Theorem 1.1) and almost simple groups $A$ (as in Theorem 1.3), as well as the extensions of $A$ by $Q$, decorations of finite simple groups. It is most likely that one can complete the picture given in the above theorems, allowing both perfect central extensions and outer automorphisms, by deducing from Theorems 1.1 and 1.3 that $B_{0}(G)=0$ for all nearly simple groups $G$ (see the definition in Section 2.3 below) excluding the groups related to the above listed exceptional cases. In particular, this statement holds true for all finite "reductive" groups such as the general linear group $G L(n, q)$, the general unitary group $G U(n, q)$, and the like.

Our notation is standard and mostly follows GLS. Throughout below "simple group" means "finite nonabelian simple group". Our proofs heavily rely on the classification of such groups.

\section{Preliminaries}

In order to make the exposition as self-contained as possible, in this section we collect the group-theoretic information needed in the proofs. All groups are assumed finite (although some of the notions discussed below can be defined for infinite groups as well).

\subsection{Schur multiplier}

The material below (and much more details) can be found in $\mathrm{Ka}$.

The group $\mathrm{M}(G):=H^{2}(G, \mathbb{Q} / \mathbb{Z})$, where $G$ acts on $\mathbb{Q} / \mathbb{Z}$ trivially, is called the Schur multiplier of $G$. It can be identified with the kernel of some central extension

$$
1 \rightarrow \mathrm{M}(G) \rightarrow \widetilde{G} \rightarrow G \rightarrow 1 .
$$

The covering group $\widetilde{G}$ is defined uniquely up to isomorphism provided $G$ is perfect (i.e., coincides with its derived subgroup $[G, G]$ ).

We will need to compute $\mathrm{M}(G)$ in the case where $G$ is a semidirect product of a normal subgroup $N$ and a subgroup $H$. If $A$ is an abelian group on which 
$G$ acts trivially, the restriction map $\operatorname{Res}_{H}: H^{2}(G, A) \rightarrow H^{2}(H, A)$ gives rise to a split exact sequence [Ka, Prop. 1.6.1]

$$
1 \rightarrow K \rightarrow H^{2}(G, A) \rightarrow H^{2}(H, A) \rightarrow 1
$$

The kernel $K$ can be computed from the exact sequence [Ka, Th. 1.6.5(ii)]

$$
1 \rightarrow H^{1}(H, \operatorname{Hom}(N, A)) \rightarrow K \stackrel{\operatorname{Res}_{N}}{\rightarrow} H^{2}(N, A)^{H} \rightarrow H^{2}(H, \operatorname{Hom}(N, A)) .
$$

If $N$ is perfect and $A=\mathbb{Q} / \mathbb{Z}$, we have $\operatorname{Hom}(N, A)=1$ and thus $[\mathrm{Ka}$, Lemma $16.3 .3]$

$$
\mathrm{M}(G) \cong \mathrm{M}(N)^{H} \times \mathrm{M}(H) .
$$

\subsection{Bogomolov multiplier}

The following properties of $B_{0}(G):=\operatorname{ker}\left[H^{2}(G, \mathbb{Q} / \mathbb{Z}) \rightarrow \prod_{A} H^{2}(A, \mathbb{Q} / \mathbb{Z})\right]$ are taken from [Bo87, $\mathrm{BMP}$.

(1) $B_{0}(G)=\operatorname{ker}\left[H^{2}(G, \mathbb{Q} / \mathbb{Z}) \rightarrow \prod_{B} H^{2}(B, \mathbb{Q} / \mathbb{Z})\right]$, where the product is taken over all bicyclic subgroups $B=\mathbb{Z}_{m} \times \mathbb{Z}_{n}$ of $G$ [Bo87, BMP, Cor. 2.3].

(2) For an abelian group $A$ denote by $A_{p}$ its $p$-primary component. We have

$$
B_{0}(G)=\bigoplus_{p} B_{0, p}(G)
$$

where $B_{0, p}(G):=B_{0}(G) \cap \mathrm{M}(G)_{p}$. For any Sylow $p$-subgroup $S$ of $G$ we have $B_{0, p}(G) \subseteq B_{0}(S)$. In particular, if all Sylow subgroups of $G$ are abelian, $B_{0}(G)=0$ [Bo87], BMP, Lemma 2.6].

(3) If $G$ is an extension of a cyclic group by an abelian group, then $B_{0}(G)=0$ Bo87, Lemma 4.9].

(4) For $\gamma \in \mathrm{M}(G)$ consider the corresponding central extension:

$$
1 \rightarrow \mathbb{Q} / \mathbb{Z} \stackrel{i}{\rightarrow} \widetilde{G}_{\gamma} \rightarrow G \rightarrow 1
$$

and denote

$$
K_{\gamma}:=\left\{h \in \mathbb{Q} / \mathbb{Z} \mid i(h) \in \bigcap_{\chi \in \operatorname{Hom}\left(\widetilde{G}_{\gamma}, \mathbb{Q} / \mathbb{Z}\right)} \operatorname{ker}(\chi)\right\} .
$$

Then $\gamma$ does not belong to $B_{0}(G)$ if and only if some nonzero element of $K_{\gamma}$ can be represented as a commutator of a pair of elements of $\widetilde{G}_{\gamma}$ [BMP, Cor. 2.4]. 
(5) If $0 \neq \gamma \in \mathrm{M}(G)$, we say that $G$ is $\gamma$-minimal if the restriction of $\gamma$ to all proper subgroups $H \subset G$ is zero. A $\gamma$-minimal group must be a $p$-group. We say that a $\gamma$-minimal nonabelian $p$-group $G$ is a $\gamma$-minimal factor if for any quotient map $\rho: G \rightarrow G / H$ there is no $\gamma^{\prime} \in B_{0}(G / H)$ such that $\gamma=\rho^{*}\left(\gamma^{\prime}\right)$ and $\gamma^{\prime}$ is $G / H$-minimal. A $\gamma$-minimal factor $G$ must be a metabelian group (i.e., $[[G, G],[G, G]]=0$ ) with central series of length at most $p$, and the order of $\gamma$ in $\mathrm{M}(G)$ equals $p$ [Bo87, Theorem 4.6]. Moreover, if $G$ is a $\gamma$-minimal $p$-group which is a central extension of $G^{\mathrm{ab}}:=G /[G, G]$ and $G^{\mathrm{ab}}=\left(\mathbb{Z}_{p}\right)^{n}$, then $n=2 m$ and $n \geq 4$ [Bo87, Lemma 5.4].

\subsection{Finite simple groups}

We need the following facts concerning finite simple groups (see, e.g., GLS]) believing that the classification of finite simple groups is complete.

(1) Classification. Any finite simple group $L$ is either a group of Lie type, or an alternating group, or one of 26 sporadic groups.

(2) Schur multipliers. As $L$ is perfect, it has a unique covering group $\widetilde{L}$, and $L \cong \widetilde{L} / \mathrm{M}(L)$. The Schur multipliers $\mathrm{M}(L)$ of all finite simple groups $L$ are given in [GLS, 6.1].

(3) Automorphisms. The group of outer automorphisms $\operatorname{Out}(L):=\operatorname{Aut}(L) / L$ is solvable. It is abelian provided $L$ is an alternating or a sporadic group. For groups of Lie type defined over a finite field $F=\mathbb{F}_{q}$ the structure of $\operatorname{Out}(L)$ can be described as follows. If $L$ comes from a simple algebraic group $\bar{L}$ defined over $\bar{F}$, we denote by $\bar{T}$ a maximal torus in $\bar{L}$. Every automorphism of $L$ is a product $i d f g$ where $i$ is an inner automorphism (identified with an element of $L$ ), $d$ is a diagonal automorphism (induced by conjugation by an element $h$ of the normalizer $N_{\bar{T}}(L)$, see [GLS, 2.5.1(b)]), $f$ is a field automorphism (arising from an automorphism of the field $\bar{F}$ ), and $g$ is a graph automorphism (induced by an automorphism of the Dynkin diagram corresponding to $L$ ); see GL, Ch. 2, $\S 7]$ or [GLS, 2.5] for more details.

The group $\operatorname{Out}(L)$ is a split extension of $\operatorname{Outdiag}(L):=\operatorname{Inndiag}(L) / L$ by the group $\Phi \Gamma$, where Inndiag $(L)$ is the group of inner-diagonal automorphisms of $L$ (generated by all $i$ 's and $d$ 's as above), $\Phi$ is the group of field automorphisms and $\Gamma$ is the group of graph automorphisms of $L$. The group $\mathcal{O}=\operatorname{Outdiag}(L)$ is isomorphic to the centre of $\widetilde{L}$ by the isomorphism preserving the action of $\operatorname{Aut}(L)$ and is nontrivial only in the following cases (where $(m, n)$ stands for the greatest common divisor of $m$ and $n$ ):

$L$ is of type $A_{n}(q) ; \mathcal{O}=\mathbb{Z}_{(n+1, q)}$; 
$L$ is of type ${ }^{2} A_{n}(q) ; \mathcal{O}=\mathbb{Z}_{(n+1, q-1)} ;$

$L$ is of type $B_{n}(q), C_{n}(q)$, or ${ }^{2} D_{2 n}(q) ; \mathcal{O}=\mathbb{Z}_{(2, q-1)}$;

$L$ is of type $D_{2 n}(q) ; \mathcal{O}=\mathbb{Z}_{(2, q-1)} \times \mathbb{Z}_{(2, q-1)}$;

$L$ is of type ${ }^{2} D_{2 n+1}(q) ; \mathcal{O}=\mathbb{Z}_{(4, q-1)}$;

$L$ is of type ${ }^{2} E_{6}(q) ; \mathcal{O}=\mathbb{Z}_{(3, q-1)}$;

$L$ is of type $E_{7}(q) ; \mathcal{O}=\mathbb{Z}_{(2, q-1)}$.

If $L$ is of type ${ }^{d} \Sigma(q)$ for some root system $\Sigma(d=1,2,3)$, the group $\Phi$ is isomorphic to $\operatorname{Aut}\left(\mathbb{F}_{q^{d}}\right)$. If $d=1$, then $\Gamma$ is isomorphic to the group of symmetries of the Dynkin diagram of $\Sigma$ and $\Phi \Gamma=\Phi \times \Gamma$ provided $\Sigma$ is simply-laced; otherwise, $\Gamma=1$ except if $\Sigma=B_{2}, F_{4}$, or $G_{2}$ and $q$ is a power of 2,2 , or 3 , respectively, in which cases $\Phi \Gamma$ is cyclic and $[\Phi \Gamma: \Phi]=2$. If $d \neq 1$, then $\Gamma=1$.

The action of $\Phi \Gamma$ on $\mathcal{O}$ is described as follows. If $L \not D_{2 n}(q)$, then $\Phi$ acts on the cyclic group $\mathcal{O}$ as $\operatorname{Aut}\left(\mathbb{F}_{q^{d}}\right)$ does on the multiplicative subgroup of $\mathbb{F}_{q^{d}}$ of the same order as $\mathcal{O}$; if $L \cong D_{2 n}(q)$, then $\Phi$ centralizes $\mathcal{O}$. If $L \cong A_{n}(q), D_{2 n+1}(q)$, or $E_{6}(q)$, then $\Gamma=\mathbb{Z}_{2}$ acts on $\mathcal{O}$ by inversion; if $L \cong D_{2 n}(q)$ and $q$ is odd, then $\Gamma$, which is isomorphic to the symmetric group $S_{3}$ (for $m=2$ ) or to $\mathbb{Z}_{2}$ (for $m>2$ ) acts faithfully on $\mathcal{O}=\mathbb{Z}_{2} \times \mathbb{Z}_{2}$.

(4) Decorations. It is often useful to consider groups close to finite simple groups, namely, quasisimple and almost simple groups, as in the statements of Theorems 1.1 and 1.3 above. As an example, if the simple group under consideration is $L=P S L(2, q)$, the group $S L(2, q)$ is quasisimple and the group $P G L(2, q)$ is almost simple. More generally, one can consider semisimple groups (central products of quasisimple groups) and nearly simple groups $G$, i.e., such that the generalized Fitting subgroup $F^{*}(G)$ is quasisimple. $F^{*}(G)$ is defined as the product $E(G) F(G)$ where $E(G)$ is the layer of $G$ (the maximal semisimple normal subgroup of $G$ ) and $F(G)$ is the Fitting subgroup of $G$ (or the nilpotent radical, i.e., the maximal nilpotent normal subgroup of $G$ ). The general linear group $G L(n, q)$ is an example of a nearly simple group.

\section{Proofs}

Proof (of Theorem [1.1). As $G$ is perfect, there exists a unique universal central covering $\widetilde{G}$ of $G$ whose centre $Z(\widetilde{G})$ is isomorphic to $\mathrm{M}(G)$ and any other perfect central extension of $G$ is a quotient of $\widetilde{G}$. So we can argue exactly as in [Bo87, Remark after Lemma 5.7] and BMP. Namely, $B_{0}(G)$ coincides with the collection of classes whose restriction to any bicyclic subgroup of $G$ is zero, see 2.2(1). Therefore, to establish the assertion of the theorem, it is enough to prove that any $z \in Z(\widetilde{G})$ can be represented as a commutator $z=[a, b]$ of some $a, b \in \widetilde{G}$. Moreover, it is enough to prove that such a representation exists for all elements $z$ of prime power order, see 2.2(2). 
It remains to apply the results of Blau [B] who classified all elements $z$ having a fixed point in the natural action on the set of conjugacy classes of $\widetilde{G}$ (such elements evidently admit a needed representation as a commutator):

Theorem 3.1. ([B1, Theorem 1]) Assume that $G$ is a quasisimple group and let $z \in Z(G)$. Then one of the following holds:

(i) $\operatorname{order}(z)=6$ and $G / Z(G) \cong A_{6}, A_{7}, F i_{22}, P S U\left(6,2^{2}\right)$, or ${ }^{2} E_{6}\left(2^{2}\right)$;

(ii) $\operatorname{order}(z)=6$ or 12 and $G / Z(G) \cong P S L(3,4), P S U\left(4,3^{2}\right)$ or $M_{22}$;

(iii) $\operatorname{order}(z)=2$ or $4, G / Z(G) \cong P S L(3,4)$, and $Z(G)$ is noncyclic;

(iv) there exists a conjugacy class $C$ of $G$ such that $C z=C$.

This theorem implies that the only possibility for an element of $G$ of prime power order to act on the set of conjugacy classes without fixed points is case (iii) where $\widetilde{G} / Z(\widetilde{G}) \cong P S L(3,4)$ and $z$ is an element of order 2 or 4 . So the classes $\gamma \in H^{2}(G, \mathbb{Q} / \mathbb{Z})$ corresponding to such $z$ 's are the only candidates for nonzero elements of $B_{0}(G)$.

A more detailed analysis of the case $P S L(3,4)$, where $Z:=Z(\widetilde{G}) \cong \mathbb{Z}_{3} \times$ $\mathbb{Z}_{4} \times \mathbb{Z}_{4}$, is sketched in $[$ Bl, Remark (2) after Theorem 1]. The result (rechecked by MAGMA computations) looks as follows: all elements of $Z$ of orders 2 and 3 fix some conjugacy class of $\widetilde{G}$, all elements of orders 6 and 12 act without fixed points, and of the twelve elements of order 4 exactly six fix a conjugacy class of $\widetilde{G}$.

First note that this description implies $B_{0}(P S L(3,4))=0$. Indeed, the criterion given in 2.2(4) can be rephrased for a quasisimple group $G$ as follows: $B_{0}(G)=0$ if and only if $Z(\widetilde{G})$ has a system of generators each of those can be represented as a commutator of a pair of elements of $\widetilde{G}$. It remains to notice that if $G=P S L(3,4)$, each 5 -tuple of elements of order 4 in $Z$ generates $\mathbb{Z}_{4} \times \mathbb{Z}_{4}$ because the subgroup of the shape $\mathbb{Z}_{4} \times \mathbb{Z}_{2}$ contains only 4 elements of order 4 (I am indebted to O. Gabber for this argument). (Another way to prove that $B_{0}(P S L(3,4))=0$ was demonstrated in BMP] where Lemma 5.3 establishes a stronger result: vanishing of $B_{0}(S)$, where $S$ is a 2-Sylow subgroup of $P S L(3,4)$.)

The only cases where the condition of the above mentioned criterion breaks down are those where $G$ is a 12 - or 4-cover of $\operatorname{PSL}(3,4)$. Indeed, in these cases we have $G=\widetilde{G} / Z$ where $Z$ is generated either by an element of order 4 (which may be not representable as a commutator) or of order 12 (which cannot be representable as a commutator). Thus in these cases we have $B_{0}(G) \neq 0$. More precisely, in both cases we have $B_{0}(G)=\mathbb{Z}_{2}$ because the subgroup generated by commutators is of index 2 in $G$ (I thank O. Gabber for this remark).

Remark 3.2. It is interesting to compare [BMP, Lemma 3.1] with a theorem from the $\mathrm{PhD}$ thesis of Robert Thompson [Th, Theorem 1]. 
Proof (of Theorem 1.3). Let $L \subseteq G \subseteq \operatorname{Aut}(L)$ where $L$ is a simple group. Clearly, it is enough to prove the theorem for $G=\operatorname{Aut}(L)$. The group $\operatorname{Out}(L)=\operatorname{Aut}(L) / L$ of outer automorphisms of $L$ acts on $\mathrm{M}(L)$, and since $L$ is perfect, we have an isomorphism

$$
\mathrm{M}(G) \cong \mathrm{M}(L)^{\operatorname{Out}(L)} \times \mathrm{M}(\operatorname{Out}(L))
$$

(see (1)).

Lemma 3.3. $B_{0}(\operatorname{Out}(L))=0$.

Proof (of Lemma 3.3). We maintain the notation of Section 2.3. If Out $(L)$ is abelian, the statement is obvious. This includes the cases where $L$ is an alternating or a sporadic group. So we may assume $L$ is of Lie type. If $\mathcal{O}=1$, i.e., $L$ is of type $E_{8}, F_{4}$, or $G_{2}$, the result follows immediately. If the group $\Phi \Gamma$ is cyclic, the result follows because $\mathcal{O}$ is abelian (see 2.3(3)). This is the case for all groups having no graph automorphisms, in particular, for all groups of type $B_{n}$ or $C_{n}(n \geq 3), E_{7}$, and for all twisted forms. For the groups of type $B_{2}$, the group $\Phi \Gamma$ is always cyclic. It remains to consider the cases $A_{n}$, $D_{n}$, and $E_{6}$. In the case $L=E_{6}$ all Sylow $p$-subgroups of $\operatorname{Out}(L)$ are abelian, and the result holds. Let $L=D_{2 m}(q)$. If $q$ is even, we have $\mathcal{O}=1, \Gamma=\mathbb{Z}_{2}$ (if $m>2$ ) or $S_{3}$ (if $m=2$ ); in both cases the Sylow $p$-subgroups of $\operatorname{Out}(L)$ are abelian, and we are done. If $q$ is odd, we have $\mathcal{O}=\mathbb{Z}_{2} \times \mathbb{Z}_{2}$, and $\Phi$ centralizes $\mathcal{O}$ (see Section 2.3), so every Sylow $p$-subgroup of $\operatorname{Out}(L)$ can be represented as an extension of a cyclic group by an abelian group, and we conclude as above. Finally, let $L$ be of type $A_{n}(q)$ or $D_{2 m+1}(q)$. Then we have $\mathcal{O}=\mathbb{Z}_{h}$, $h=(n+1, q-1)$ or $h=(4, q-1)$, respectively, $\Gamma=\mathbb{Z}_{2}, \Phi=\operatorname{Aut}\left(\mathbb{F}_{q}\right)$. The action of both $\Gamma$ and $\Phi$ on $\mathcal{O}$ may be nontrivial: $\Gamma$ acts by inversion, $\Phi$ acts on $\mathcal{O}$ as $\operatorname{Aut}\left(\mathbb{F}_{q}\right)$ does on the multiplicative subgroup of $\mathbb{F}_{q}$ of the same order as $\mathcal{O}$. Hence we can represent the metabelian group $\operatorname{Out}(L)$ in the form

$$
1 \rightarrow V \rightarrow \operatorname{Out}(L) \rightarrow A \rightarrow 1,
$$

where $V$, the derived subgroup of $\operatorname{Out}(L)$, is isomorphic to a cyclic subgroup $\mathbb{Z}_{c}$ of $\mathcal{O}$, and the abelian quotient $A$ is of the form $\mathbb{Z}_{a} \times \mathbb{Z}_{b} \times \mathbb{Z}_{2}$ for some integers $a, b, c$. Since it is enough to establish the result for a Sylow 2-subgroup, we may assume that $a, b$ and $c$ are powers of 2 . Then the statement of the lemma follows from the properties of $\gamma$-minimal elements described in Section 2.2. Indeed, if $\gamma$ is a nonzero element of $B_{0}(G)$ and $G$ is $\gamma$-minimal, then $G$ is metabelian, both $V$ and $A$ are of exponent $p$, and in any representation of $G$ in the form (3) the group $A$ must have an even number $s=2 t$ of direct summands $\mathbb{Z}_{p}$ with $t \geq 2$. However, if $G$ is a Sylow 2-subgroup of $\operatorname{Out}(L)$, this is impossible because $A$ contains only three direct summands. Thus $B_{0}\left(S y l_{2}(\operatorname{Out}(L))\right)=0$, and so $B_{0}(\operatorname{Out}(L))=0$. The lemma is proved.

We can now finish the proof of the theorem. Let $\gamma$ be a nonzero element of $B_{0}(G)$. Using the isomorphism (2), we can represent $\gamma$ as a pair $\left(\gamma_{1}, \gamma_{2}\right)$ 
where $\gamma_{1} \in \mathrm{M}(L), \gamma_{2} \in \mathrm{M}(\operatorname{Out}(L))$. Restricting to the bicyclic subgroups of $G$, we see that $\gamma_{1} \in B_{0}(L), \gamma_{2} \in B_{0}(\operatorname{Out}(L))$, and the result follows from Theorem 1.1 and Lemma 3.3 .

Acknowledgements. The author's research was supported in part by the Minerva Foundation through the Emmy Noether Research Institute of Mathematics, the Israel Academy of Sciences grant 1178/06, and a grant from the Ministry of Science, Culture and Sport (Israel) and the Russian Foundation for Basic Research (the Russian Federation). This paper was mainly written during the visit to the MPIM (Bonn) in August-September 2007 and completed during the visit to ENS (Paris) in April-May 2008. The support of these institutions is highly appreciated.

My special thanks are due to O. Gabber who noticed a gap in an earlier version of the paper and helped to fill it. I am also grateful to M. Conder, D. Holt and A. Hulpke for providing representations of $P S L(3,4)$ necessary for MAGMA computations, and to N. A. Vavilov for useful correspondence.

\section{References}

Bl. H. I. BLAU, A fixed-point theorem for central elements in quasisimple groups, Proc. Amer. Math. Soc. 122 (1994) 79-84.

Bo87. F. A. Bogomolov, The Brauer group of quotient spaces by linear group actions, Izv. Akad. Nauk. SSSR Ser. Mat. 51 (1987) 485-516; English transl. in Math. USSR Izv. 30 (1988) 455-485.

Bo92. F. A. Bogomolov, Stable cohomology of groups and algebraic varieties, Mat. Sb. 183 (1992) 1-28; English transl. in Sb. Math. 76 (1993) 1-21.

Bo07. F. Bogomolov, Stable cohomology of finite and profinite groups, "Algebraic Groups" (Y. Tschinkel, ed.), Universitätsverlag Göttingen, 2007, 19-49.

BMP. F. Bogomolov, J. Maciel, T. Petrov, Unramified Brauer groups of finite simple groups of Lie type $A_{\ell}$, Amer. J. Math. 126 (2004) 935-949.

CTS. J.-L. Colliot-ThÉLÈne, J.-J. SAnsuc, The rationality problem for fields of invariants under linear algebraic groups (with special regards to the Brauer group), Proc. Intern. Colloquium on Algebraic Groups and Homogeneous Spaces (Mumbai 2004) (V. Mehta, ed.), TIFR Mumbai, Narosa Publishing House, 2007, 113-186.

GS. P. Gille, T. Szamuely, Central Simple Algebras and Galois Cogomology, Cambridge Univ. Press, Cambridge, 2006.

GL. D. Gorenstein, R. Lyons, The Local Structure of Finite Groups of Characteristic 2 Type, Mem. Amer. Math. Soc., vol. 276, Providence, RI, 1983.

GLS. D. Gorenstein, R. Lyons, R. Solomon, The Classification of the Finite Simple Groups, Number 3, Math. Surveys and Monographs, vol. 40, no. 3, Amer. Math. Soc., Providence, RI, 1998.

Ka. G. Karpilovsky, Group Representations, vol. 2, North-Holland Math. Studies 177, North-Holland, Amsterdam et al., 1993.

Sa. D. J. SAltman, Noether's problem over an algebraically closed field, Invent. Math. 77 (1984) 71-84.

Sh. I. R. Shafarevich, The Lüroth problem, Trudy Mat. Inst. Steklov 183 (1990) 199 204; English transl. Proc. Steklov Inst. Math. 183 (1991) 241-246. 
Th. R. C. Thompson, Commutators in the special and general linear groups, Trans. Amer. Math. Soc. 101 (1961) 16-33. 